\title{
ON LIE SEMI-GROUPS
}

\author{
R. P. LANGLANDS
}

1. Suppose we have a semi-group structure defined on

$$
\pi=\left\{\left(p^{1}, \ldots, p^{n}\right) \mid p^{1} \geqslant 0, \ldots, p^{n} \geqslant 0\right\},
$$

a subset of real Euclidean $n$-space, $E_{n}$, by $(p, q) \rightarrow F(p, q)=p \circ q$. In this note we shall be concerned with a representation $T($.$) of \pi$ as a semi-group of bounded linear operators on a Banach space $\mathfrak{X}$. More particularly, we suppose that postulates $P_{1}, P_{2}, P_{3}, P_{5}$, and $P_{6}$ of chapter 25 of (2) are satisfied so that, by Theorem 25.3 .1 of that book, there is a continuous function, $f($.$) ,$ defined on $\pi$ such that $f((\rho+\sigma) a)=f(\rho a) \circ f(\sigma a)$ for $a \in \pi, \rho, \sigma \geqslant 0$; that the representation is strongly continuous in a neighbourhood of the origin and that $T(0)=I$. Then for $a \in \pi, \rho \rightarrow T(f(\rho a))$ is a strongly continuous one parameter semi-group; denote its infinitesimal generator by $A(a)$. We shall study, under the assumption that $F(p, q)$ is three times continuously differentiable, the relations among the $A(a)$ and their adjoints $A^{*}(a)$. Following a suggestion of Hille (1) we first prove some "Dense Graph Theorems." Using these we show that the expected linear and commutation relations hold. We also show that $\bigcap_{a \in \pi} \mathfrak{D}(A(a))[\mathfrak{D}(A(a))$ is the domain of $A(a)$ ] is invariant under $T(p)$ for small $p$ in $\pi$. The proofs are so formulated that, with minor changes, they remain valid in other situations.

We should like to thank C. T. Ionescu Tulcea who suggested that the methods of (3) might be applicable to the questions discussed here.

2. We set

$$
\begin{gathered}
\frac{\partial F^{k}}{\partial p^{j}}=F_{j ;}^{k}(p, q) ; \frac{\partial F^{k}}{\partial q^{j}}=F_{; j}^{k}(p, q) ; \frac{\partial^{2} F^{k}}{\partial q^{i} \partial p^{j}}=F_{j ; i}^{k}(p, q) ; \\
F_{i ; j}^{k}(0,0)-F_{j ; i}^{k}(0,0)=\gamma_{i j}^{k} .
\end{gathered}
$$

$F(p, q)$ may be extended to a twice continuously differentiable function defined on $E_{n} \times E_{n}$. Denote some fixed extension by $F(q, p)$. Since

$$
F_{j ;}^{k}(0,0)=F_{; j}^{k}(0,0)=\delta_{j}^{k}
$$

there are open spheres $N_{1}, N_{2} \subseteq N_{1}$ about the origin and three times continously differentiable functions $\psi(q, h)$ and $\chi(q, h)$ defined on $N_{1} \times N_{1}$ such that $\psi(0,0)=\chi(0,0)=0, F(h, \psi(q, h))=q$, and $F(\chi(q, h), h)=q$. Moreover if $F(h, p)=q[F(p, h)=q]$ with $p, h \in N_{2}$, then $q \in N_{1}$ and $\psi(q, h)=$ $p[\chi(q, h)=p]$. We may also suppose that all derivatives of $\psi(q, h)$ and $\chi(q, h)$ up to the third order are bounded in $N_{1}$, that $T($.$) is strongly con-$

Received June 30, 1959. 
tinuous in $N_{1} \cap \pi$, and that $\operatorname{det}\left(F_{j ;}{ }^{k}(0, p)\right) \geqslant 1 / 2$ and $\operatorname{det}\left(F_{; j}{ }^{k}(p, 0)\right) \geqslant 1 / 2$ for $p$ in $N_{1}$. If $N \subseteq N_{1}$ is an open sphere about the origin, set

$$
E(N)=\left\{y=\int_{\pi} K(q) T(q) x d q \mid x \in \mathfrak{X}, K(q) \in C^{2}(N \cap \pi)\right\}
$$

$C^{2}(N \cap \pi)$ is the set of twice continuously differentiable functions which are 0 outside of $N \cap \pi . E(N)$ is dense in $\mathfrak{X}$.

Proposition 1. Let $N_{3}$ be an open sphere about the origin with $F\left(N_{3}, N_{3}\right) \subseteq$ $N_{2}$. If $y \in E\left(N_{3}\right)$, then $T(p) y$ is a twice continuously differentiable function of $p$ in $N_{3} \cap \pi$.

Proof. We understand that some derivatives at the boundary will be one-sided. If $y \in E\left(N_{3}\right)$ and $e_{j}=\left(\delta_{j}{ }^{1}, \ldots, \delta_{j}{ }^{n}\right)$ we have, recalling that $K(q)$ is 0 outside of $N_{3} \cap \pi$,

$$
\begin{aligned}
\lim _{s \rightarrow 0} & s^{-1}\left(T\left(p+s e_{j}\right) y-T(p) y\right) \\
& =\lim _{s_{\rightarrow}} s^{-1} \int_{N_{3} \cap_{\pi}} K(q)\left(T\left(\left(p+s e_{j}\right) \circ q\right)-T(p \circ q)\right) x d q \\
& =\left.\lim _{s \rightarrow 0} s^{-1} \int_{N_{2} \cap_{\pi}}\left(K(\psi(q, r)) \operatorname{det}\left(\frac{\partial \psi^{k}}{\partial q^{i}}(q, r)\right)\right)\right|_{r=p} ^{r=p+s e_{j}} T(q) x d q \\
& =\int_{N_{2} \cap_{\pi}} \frac{\partial}{\partial p^{j}}\left(K(\psi(q, p)) \operatorname{det}\left(\frac{\partial \psi^{k}}{\partial q^{i}}(q, p)\right)\right) T(q) x d q+\lim _{s \rightarrow 0} \int_{N_{2} \cap_{\pi}} G(q, p, s) d q \\
& =\int_{N_{2} \cap_{\pi}} \frac{\partial}{\partial p^{j}}\left(K(\psi(q, p)) \operatorname{det}\left(\frac{\partial \psi^{k}}{\partial q^{i}}(q, p)\right)\right) T(q) x d q
\end{aligned}
$$

since $G(q, p, s)$ converges boundedly to 0 with $s$. The final integral is a continuous function of $p$. In a similar manner we show that it is once continuously differentiable.

We remark the following formulae, valid for $y \in E\left(N_{3}\right), p \in N_{3} \cap \pi$ :

$$
\begin{aligned}
& \text { (i) } \lim _{s \rightarrow 0} s^{-1}(T(f(s a))-I) T(p) y=\lim _{s \rightarrow 0} s^{-1}(T(f(s a) \circ p) y-T(p) y) \\
& =\lim _{s \rightarrow 0}\left[\sum_{j=1}^{n} s^{-1}\left(F^{j}(f(s a), p)-p^{j}\right) \frac{\partial}{\partial p^{j}} T(p) y+s^{-1} o(|f(s a) \circ p-p|)\right] \\
& \text { (1) } \quad=\sum_{j=1}^{n}\left(\sum_{i=1}^{n} F_{i ;}^{j}(0, p) a^{i}\right) \frac{\partial}{\partial p^{j}} T(p) y .
\end{aligned}
$$

So $T(p) y \in \mathfrak{D}(A(a))$ and $A(a) T(p) y$ is given by $(1)$.

$$
\begin{aligned}
T(p) A(a) y & =\lim _{s \rightarrow 0} s^{-1}(T(p \circ f(s a)) y-T(p) y) \\
& =\sum_{j=1}^{n}\left(\sum_{k=1}^{n} F_{; k}^{j}(p, 0) a^{k}\right) \frac{\partial}{\partial p^{j}} T(p) y .
\end{aligned}
$$

(iii) Setting $\left(\gamma_{k}^{j}(p)\right)=\left(F^{j} ;{ }_{k}(p, 0)\right)^{-1}$, 


$$
\frac{\partial}{\partial p^{j}} T(p) y=\sum_{k=1}^{n} \gamma_{k}^{j}(p) T(p) A\left(e_{k}\right) y \text {. }
$$

(4) $A(a) A(b) T(p) y=\sum_{k, j=1}^{n}\left(\sum_{i=1}^{n} \beta_{i}^{k}(p) b^{i}\right)\left(\sum_{m=1}^{n} F_{m ;}^{j}(0, p) a^{m}\right) \frac{\partial}{\partial p^{j}} T(p) A\left(e_{k}\right) y$.

(v) $(\alpha) \quad A(a+b) y=A(a) y+A(b) y$

(в) $A\left(e_{i}\right) A\left(e_{j}\right) y-A\left(e_{j}\right) A\left(e_{i}\right) y=\sum_{k=1}^{n} \gamma_{i j}^{k} A\left(e_{k}\right) y$.

For a proof of the latter relation, see (2, p. 758).

3. We come now to the "Dense Graph Theorems."

TheOrem 1. Let $\left\{a_{1}, \ldots, a_{p}\right\} \subseteq \pi$; if $G_{0}$ is the closure in the product topology on $X x \ldots x X(p+1$ factors $)$ of $\left\{\left(x, A\left(a_{1}\right) x, \ldots, A\left(a_{p}\right) x\right) \mid x \in E\left(N_{3}\right)\right\}$ and

$$
G=\left\{\left(x, A\left(a_{1}\right) x, \ldots, A\left(a_{p}\right) x\right) \mid x \in \bigcap_{j=1}^{p} \mathfrak{D}\left(A\left(a_{j}\right)\right)\right\}
$$

then $G_{0}=G$.

Proof. $G \supseteq G_{0}$ since an infinitesimal generator is a closed operator. We show that $G_{0} \supseteq G$. Let $\left\{b_{r+1}, \ldots, b_{n}\right\}$ be a maximal linearly independent subset of $\left\{a_{1}, \ldots, a_{p}\right\}$; it is sufficient to prove the theorem for the former set. Let $\left\{b_{1}, \ldots, b_{n}\right\} \subseteq \pi$ be a basis for $E_{n}$. If $t=\left(t^{1}, \ldots, t^{n}\right) \in \pi$, set $p(t)=$ $f\left(t^{1} b_{1}\right) \circ \ldots \circ f\left(t^{n} b_{n}\right) \cdot p(t)$ is a twice continuously differentiable map of $\pi$ into $\pi$ and may be extended to a twice continuously differentiable map of $E_{n}$ into $E_{n}$. Since

$$
\frac{\partial p^{k}}{\partial t^{j}}(0)=b_{j}^{k}
$$

$p(t)$ has a twice continuously differentiable inverse defined in a sphere $\mathrm{N}_{4}$ about the origin. We may suppose that $F\left(N_{4}, N_{4}\right) \subseteq N_{3}$ and that all derivatives of the inverse function up to the second order are bounded in $N_{4}$. If $y \in E\left(N_{4}\right)$ and $p \in N_{4} \cap \pi$ then $T(p) y \in E\left(N_{3}\right)$. For $y \in E\left(N_{4}\right)$, set

$$
u(y, s)=\left(\prod_{j=1}^{n} s^{j}\right)^{-1} \int_{R(s)} S(t) y d t
$$

where $s=\left(s^{1}, \ldots, s^{n}\right), S(t)=T(p(t)), R(s)$ is the rectangle with sides $\left[0, s^{j} e_{j}\right]$, and $R(s)$ is contained in the image of $N_{4}$ under the inverse map. Using (1)

$$
\begin{aligned}
A\left(b_{k}\right) u(y, s) & =\left(\prod_{j=1}^{n} s^{j}\right)^{-1} \int_{R(s)} A\left(b_{j}\right) S(t) y d t \\
& =\left(\prod_{j=1}^{n} s^{j}\right)^{-1} \int_{R(s)} \sum_{i=1}^{n} \zeta_{k}^{i}(t) \frac{\partial}{\partial t^{i}} S(t) y d t
\end{aligned}
$$


where

$$
\zeta_{k}^{i}(t)=\sum_{j, m=1}^{n} F_{m}^{j}:(0, p(t)) b_{k}^{m} \frac{\partial t^{i}}{\partial p^{j}}
$$

is once continuously differentiable. Integrating by parts,

$$
\begin{aligned}
A\left(b_{k}\right) u(y, s) & =\left(\prod_{j=1}^{n} s^{j}\right)^{-1}\left[\sum_{i=1}^{n} \int_{R\left(\hat{s}^{i}\right)} \zeta_{k}^{i}(t) S(t) y \mid \begin{array}{c}
\left(\begin{array}{l}
\left.\hat{t}^{i}, s^{i}\right) \\
\left(\hat{t}^{i}, 0\right.
\end{array}\right) \\
-\hat{t}^{i} .
\end{array}\right. \\
& \left.-\int_{R(s)} \sum_{i=1}^{n} \frac{\partial \zeta_{k}^{i}}{\partial t^{i}}(t) S(t) y d t\right] .
\end{aligned}
$$

Since the integral of a function with values lying in a closed subspace of a Banach space is contained in that subspace

$$
\left(u(y, s), A\left(b_{r+1}\right) u(y, s), \ldots, A\left(b_{n}\right) u(y, s)\right) \in G_{0} .
$$

Since (4) is a continuous function of $y$ and $E\left(N_{4}\right)$ is dense in $\mathfrak{X}$; for any $y \in \mathfrak{X}$, $u(y, s) \in \cap{ }_{j=1}^{n} \mathfrak{D}\left(A\left(b_{j}\right)\right)$ and (4) and (5) hold. To complete the proof it is sufficient to show

$$
\lim _{\sigma \rightarrow 0} u(y, s(\sigma)) \equiv y .
$$

$$
\lim _{\sigma \rightarrow 0} A\left(b_{k}\right) u(y, s(\sigma))=A\left(b_{k}\right) y
$$

for $k \geqslant r+1, y \in \cap_{k=r+1}^{n} \mathfrak{D}\left(A\left(b_{k}\right)\right)$, and $s(\sigma)=(\sigma, \ldots, \sigma)$. (6) is clear; to prove $(7)$ we expand $\zeta_{k}{ }^{i}(t)$ in a Taylor's series and consider

$$
\begin{aligned}
\lim _{\sigma \rightarrow 0} \sigma^{-n} & \left.\int_{R\left(\hat{s}^{i}(\sigma)\right)} \zeta_{k}^{i}(t) S(t) y \mid \begin{array}{l}
\left(\hat{t}^{i}, \sigma\right) \\
\left(\hat{t}^{i}, 0\right)
\end{array}\right) \hat{t}^{i} \\
= & \lim _{\sigma \rightarrow 0}\left[\sigma^{-n+1} \int_{R\left(\hat{s}^{i}\right)} \delta_{k}^{i} \sigma^{-1}\left(S\left(\hat{t}^{i}, \sigma\right) y-S\left(\hat{t}^{i}, 0\right) y\right) d \hat{t}^{i}+\right. \\
& +\sigma^{-n+1} \int_{R\left(\hat{s}^{i}\right)} \frac{\partial \zeta_{k}^{i}}{\partial t^{i}}(0) S\left(\hat{t}^{i}, \sigma\right) y d \hat{t}^{i}+ \\
& \left.+\sigma^{-n+1} \int_{R\left(\hat{s}^{i}\right)}\left(\sum_{j \neq i} \sigma^{-1} t^{j} \frac{\partial \zeta_{k}^{i}}{\partial t^{j}}(0)\right)\left(S\left(\hat{t}^{i}, \sigma\right) y-S\left(\hat{t}^{i}, 0\right) y\right) d \hat{t}^{i}+o(1)\right] \\
= & \delta_{k}^{i} A\left(b_{k}\right) y+\frac{\partial \zeta_{k}^{i}}{\partial t^{i}}(0) y
\end{aligned}
$$

provided

$$
\lim _{\sigma \rightarrow 0} \sigma^{-1}\left(S\left(\hat{t}^{k}, \sigma\right) y-S\left(\hat{t}^{k}, 0\right) y\right)=A\left(b_{k}\right) y .
$$

But the left side equals

$$
\begin{aligned}
& \prod_{j=1}^{k-1} T\left(f\left(t^{j} b_{j}\right)\right)\left[\sigma^{-1}\left(T\left(f\left(\sigma b_{k}\right)\right) y-y\right)+\right. \\
& \left.+\left(T\left(f\left(\sigma b_{k}\right)\right)-I\right)\left(\sum_{i=k+1}^{n} \prod_{m=k+1}^{i-1} T\left(f\left(t^{m} b_{m}\right)\right) \sigma^{-1}\left(T\left(f\left(t^{i} b_{i}\right)\right) y-y\right)\right)\right]
\end{aligned}
$$


and (8) follows if we recall that $t^{i} \leqslant \sigma$ and that $y \in \mathfrak{D}\left(A\left(b_{i}\right)\right)$ for $i \geqslant k \geqslant$ $r+1$. Summing over $i$ and taking the last term of (4) into account we obtain (7).

THEOREM 2. If $F_{0}$ is the closure in the product topology of

$$
\left\{\left(y, A\left(e_{1}\right) y, \ldots, A\left(e_{n}\right) y, A\left(e_{i}\right) A\left(e_{j}\right) y\right) \mid y \in E\left(N_{3}\right)\right\}
$$

and if

$F=$

$\left\{\left(y, A\left(e_{1}\right) y, \ldots, A\left(e_{n}\right) y, A\left(e_{i}\right) A\left(e_{j}\right) y\right) \mid y \in \bigcap_{k=1}^{n} \mathfrak{D}\left(A\left(e_{k}\right)\right) \cap \mathfrak{D}\left(A\left(e_{i}\right) A\left(e_{j}\right)\right)\right\}$,

then $F=F_{0}$.

Proof. $F$ is a closed set and thus $F \supseteq F_{0}$. We show $F_{0} \supseteq F$. Taking $b_{k}=e_{k}$ we use the notation of the proof of Theorem 1. For $y \in E\left(N_{4}\right)$

$$
\begin{aligned}
A\left(e_{i}\right) A\left(e_{j}\right) u(y, s) & =\left(\prod_{r=1}^{n} s^{r}\right)^{-1} \int_{R(s)} A\left(e_{i}\right) A\left(e_{j}\right) S(t) y d t \\
& =\left(\prod_{r=1}^{n} s^{r}\right)^{-1} \int_{R(s)} \sum_{k, m=1}^{n} \delta_{m}^{k}(t) \frac{\partial}{\partial t^{m}}\left(S(t) A\left(e_{k}\right) y\right) d t
\end{aligned}
$$

where

$$
\delta_{m}^{k}(t)=\sum_{r=1}^{n} \beta_{j}^{k}(p(t)) F_{i ;}^{r}(0, p(t)) \frac{\partial t^{m}}{\partial p^{r}}
$$

is once continuously differentiable. Integrating by parts

$$
\begin{aligned}
& A\left(e_{i}\right) A\left(e_{j}\right) u(y, s)= \\
& \quad=\left(\prod_{r=1}^{n} s^{\tau}\right)^{-1}\left[\sum_{m=1}^{n} \int_{R\left(\hat{s}^{m}\right)} \sum_{k=1}^{n} \delta_{m}^{k}(t) S(t) A\left(e_{k}\right) y \mid \begin{array}{c}
\left(\hat{t}^{m}, s^{m}\right) \\
\left.\hat{t}^{m}, 0\right)
\end{array}\right) \hat{d t^{m}} \\
& \left.\quad-\int_{R(s)} \sum_{k, m=1}^{n} \frac{\partial \delta_{m}^{k}}{\partial t^{m}}(t) S(t) A\left(e_{k}\right) y d t\right] .
\end{aligned}
$$

Theorem 1 implies that (9) holds for $y \in \cap^{n}{ }_{k=1} \mathfrak{D}\left(A\left(e_{k}\right)\right)$. The proof is then completed as above.

4. We now consider the adjoints of the infinitesimal generators. If $y^{*} \in \mathfrak{X}^{*}$ we denote the value of $y^{*}$ at $y \in \mathfrak{X}$ by $\left\langle y, y^{*}\right\rangle$. If $N \subseteq N_{1}$, set

$$
E^{*}(N)=\left\{y^{*} \in X^{*} \mid\left\langle y, y^{*}\right\rangle=\int_{\pi}\left\langle y, K(q) T^{*}(q) x^{*}\right\rangle d q,\right.
$$

with $x^{*} \in \mathfrak{X}^{*}$ and $\left.K(q) \in C^{2}(N \cap \pi)\right\}, E^{*}(N)$ is dense in $\mathfrak{X}^{*}$ in the weak* topology.

Proposition 2. If $y^{*} \in E^{*}\left(N_{3}\right), T^{*}(p) y$ is twice continuously differentiable in the weak topology for $p$ in $N_{3} \cap \pi$. 
Proof. We merely sketch the calculations since the proof is essentially the same as that of Proposition 1.

$$
\begin{aligned}
\lim _{s \rightarrow 0} s^{-1} & \int_{\pi}\left\langle y, K(q)\left(T^{*}\left(p+s e_{j}\right)-T^{*}(p)\right) T^{*}(q) x^{*}\right\rangle d q= \\
& =\lim _{s \rightarrow 0} s^{-1} \int_{\pi}\left\langle y, K(q)\left(T^{*}\left(q \circ\left(p+s e_{j}\right)\right)-T^{*}(q \circ p)\right) x^{*}\right\rangle d q \\
& =\int_{N_{2} \cap_{\pi}}\left\langle y, \frac{\partial}{\partial p^{j}}\left(K(\chi(q, p)) \operatorname{det}\left(\frac{\partial \chi^{k}}{\partial q^{i}}(q, p)\right)\right) T^{*}(q) x^{*}\right\rangle d q
\end{aligned}
$$

The last integral is again a continuously differentiable function of $p$.

We remark the following, valid for $y^{*} \in E^{*}\left(N_{3}\right)$ and $p \in N_{3} \cap \pi$ :

(i) $\lim _{s \rightarrow 0} s^{-1}\left\langle y,\left(T^{*}(f(s a))-I\right) T^{*}(p) y^{*}\right\rangle=$

$$
=\sum_{j=1}^{n}\left(\sum_{m=1}^{n} F_{; m}^{j}(p, 0) a^{m}\right) \frac{\partial}{\partial p^{j}}\left\langle y, T^{*}(p) y^{*}\right\rangle .
$$

This implies that $T^{*}(p) y^{*} \in \mathfrak{D}\left(A^{*}(a)\right)$ and that $\left\langle y, A^{*}(a) T^{*}(p) y^{*}\right\rangle$ is given by the right side of $(10)$.

(ii) As in the remarks following Proposition 1 we may show

$$
\begin{aligned}
& \left(\alpha^{\prime}\right) A^{*}(a+b) y^{*}=A^{*}(a) y^{*}+A^{*}(b) y^{*} \\
& \left(\beta^{\prime}\right) A^{*}\left(e_{i}\right) A^{*}\left(e_{j}\right) y^{*}-A^{*}\left(e_{j}\right) A^{*}\left(e_{i}\right) y^{*}=-\sum_{k=1}^{n} \gamma_{i j}^{k} A^{*}\left(e_{k}\right) y^{*} .
\end{aligned}
$$

THEOREM 3. Let $\left\{a_{1}, \ldots, a_{p}\right\} \subseteq \pi$, if $H_{0}$ is the closure in the product of the weak* topologies of $\left\{\left(y^{*}, A^{*}\left(a_{1}\right) y^{*}, \ldots, A^{*}\left(a_{p}\right) y^{*}\right) \mid y^{*} \in E^{*}\left(N_{3}\right)\right\}$ and $H=\left\{\left(y^{*}, A^{*}\left(a_{1}\right) y^{*}, \ldots, A^{*}\left(a_{p}\right) y^{*}\right) \mid y^{*} \in \cap_{i=1}^{p \mathfrak{D}}\left(A\left(a_{\jmath}\right)\right)\right\}$ then $H=H_{0}$.

Proof. $H \supseteq H_{0}$ since $A^{*}(a)$ is closed in the weak* topology. We show $H_{0} \supseteq H$. Let $\left\{b_{1}, \ldots, b_{r}\right\}$ be a maximal linearly independent subset of $\left\{a_{1}, \ldots, a_{p}\right\}$; it is sufficient to prove the theorem for the former set. Let $\left\{b_{1}, \ldots, b_{n}\right\} \subseteq \pi$ be a basis for $E_{n}$. Again we use the notation of the proof of Theorem 1. If $y^{*} \in E^{*}\left(N_{4}\right)$ set

$$
\left\langle y, u\left(y^{*}, s\right)\right\rangle=\left(\prod_{j=1}^{n} s^{j}\right)^{-1} \int_{R(s)}\left\langle y, S^{*}(t) y^{*}\right\rangle d t
$$

with $S^{*}(t)=T^{*}(p(t))$. As above

$$
\begin{aligned}
& \left\langle y, A^{*}\left(b_{k}\right) u\left(y^{*}, s\right)\right\rangle= \\
& \quad\left(\prod_{j=1}^{n} s^{j}\right)^{-1}\left[\sum_{i=1}^{n} \int_{R(\hat{s} i)} \xi_{k}^{i}(t)\left\langle y, S^{*}(t) y^{*}\right\rangle \mid \begin{array}{c}
\left.\hat{t}^{i}, s^{i}\right) \\
\left(\hat{t}^{i}, 0\right)
\end{array}\right) \hat{t}^{i} \\
& \quad-\int_{R(s)} \sum_{i=1}^{n} \frac{\partial \xi_{k}^{i}}{\partial t^{i}}(t)\left\langle y, S^{*}(t) y^{*}\right\rangle d t
\end{aligned}
$$

with

$$
\xi_{k}^{i}(t)=\sum_{j, m=1}^{n} F_{; j}^{m}(p(t), 0) b_{k}^{j} \frac{\partial t^{i}}{\partial p^{m}}
$$


As above, $u\left(y^{*}, s\right) \in \cap_{k=1}^{n} \mathfrak{D}\left(A^{*}\left(b_{k}\right)\right)$ for all $y^{*} \in \mathfrak{X}^{*}$ and $A^{*}\left(b_{k}\right) u\left(y^{*}, s\right)$ is given by (11). Moreover,

$$
\left(u\left(y^{*}, s\right), A^{*}\left(b_{1}\right) u\left(y^{*}, s\right), \ldots, A^{*}\left(b_{r}\right) u\left(y^{*}, s\right)\right) \in H_{0} .
$$

The proof may be completed as before if we show that

$$
\lim _{\sigma \rightarrow 0} \sigma^{-1}\left\langle y,\left(S^{*}\left(\hat{t^{k}}, \sigma\right)-S^{*}\left(\hat{t^{k}}, 0\right)\right) y^{*}\right\rangle=\left\langle y, A^{*}\left(b_{k}\right) y^{*}\right\rangle
$$

for $1 \leqslant k \leqslant r, t^{j} \leqslant \sigma$, and $y^{*} \in \cap_{i=1}^{r} \mathfrak{D}\left(A^{*}\left(b_{i}\right)\right)$. But the expression on the left equals

$$
\begin{gathered}
\left\langle\prod_{j=k+1}^{n} T\left(f\left(t^{j} b_{j}\right)\right) y, \sigma^{-1}\left(T^{*}\left(f\left(\sigma b_{k}\right)\right)-I\right) y^{*}\right\rangle \\
+\sum_{i=1}^{k-1}\left\langle\prod_{m=i+1}^{k-1} T\left(f\left(t^{m} b_{m}\right)\right)\left(T\left(f\left(\sigma b_{k}\right)\right)-I\right)\right. \\
\left.\prod_{j=k+1}^{n} T\left(f\left(t^{j} b_{j}\right)\right) y, \sigma^{-1}\left(T^{*}\left(f\left(t^{i} b_{i}\right)\right)-I\right) y^{*}\right\rangle ;
\end{gathered}
$$

and (12) follows since, see (3), $\sigma^{-1}\left(T^{*}\left(f\left(t^{i} b_{i}\right)\right)-I\right) y^{*}$ is uniformly bounded and $\sigma^{-1}\left(T^{*}\left(f\left(\sigma b_{k}\right)\right)-I\right) y^{*}$ converges in the weak* topology to $A^{*}\left(b_{k}\right) y^{*}$.

5. If $a=\left(a^{1}, \ldots, a^{n}\right) \in E_{n}, \quad A(a) y=\sum_{j=1}^{n} a^{j} A\left(e_{\jmath}\right) y$ is defined for $y \in E\left(N_{3}\right)$. By the remarks after Proposition $2, E^{*}\left(N_{3}\right)$ is contained in the domain of its adjoint so that $A(a)$ has a least closed extension which we again denote by $A(a)$. This notation is consistent with that used previously.

LEMMA. $A^{*}(a)$, the adjoint of $A(a)$, is the weak* closure of the operator $\sum_{j=1}^{n} a^{j} A^{*}\left(e_{j}\right)$ with domain $E^{*}\left(N_{3}\right)$.

Proof. Suppose $\left\langle y, x_{1}^{*}\right\rangle=\left\langle A(a) y, x_{2}^{*}\right\rangle$ for all $y \in E\left(N_{3}\right)$. Then, using Theorem 1 and the notation of its proof with $b_{j=} e_{j}$, for $y \in \mathfrak{X}$

$$
\begin{gathered}
\sigma^{-n} \int_{R(s(\sigma))}\left\langle S(t) y, x_{1}^{*}\right\rangle d t=\sigma^{-n} \sum_{j=1}^{n} a^{j}\left[\sum_{i=1}^{n} \int_{R\left(\hat{s}^{i}\right)}\left\langle\zeta_{j}^{i}(t) S(t) y, x_{2}^{*}\right\rangle \mid \begin{array}{c}
\left.\hat{t}^{i}, \sigma\right) \\
\left(\hat{t}^{i}, 0\right)
\end{array}\right) d \hat{t}^{i} \\
\left.-\int_{R(s)}\left(\sum_{i=1}^{n} \frac{\partial \zeta_{j}^{i}}{\partial t^{i}}(t)\right)\left\langle S(t) y, x_{2}^{*}\right\rangle d t\right] .
\end{gathered}
$$

Transposing and taking limits

$$
\lim _{\sigma \rightarrow 0} \sigma^{-n} \sum_{j=1}^{n} a^{j} \int_{R\left(\hat{s^{j}}\right)}\left\langle y,\left(S^{*}\left(\hat{t}^{j}, \sigma\right)-S^{*}\left(t^{j}, 0\right)\right) x_{2}^{*}\right\rangle \hat{d t^{j}}=\left\langle y, x_{1}^{*}\right\rangle .
$$

Then, using (11),

$$
\lim _{\sigma \rightarrow 0}\left\langle y, \sum_{j=1}^{n} a^{j} A^{*}\left(e_{j}\right) u\left(x_{2}^{*}, s(\sigma)\right)\right\rangle=\left\langle y, x_{1}^{*}\right\rangle .
$$

The lemma is now an easy consequence of Theorem 3. 
By Theorem 25.8.1 of (2) the $\gamma_{i j}{ }^{k}$ may be used to define a Lie algebra $\mathfrak{A}$ over $E_{n}$. We denote the Lie product of $a$ and $b$ by $[a, b]$. The following theorem can easily be proved using the lemma, formulae $(\alpha),(\beta),\left(\alpha^{\prime}\right)$, and $\left(\beta^{\prime}\right)$, and the Hahn-Banach theorem.

Theorem 4.I. The function $a \rightarrow A(a)$ defined on $\mathfrak{A}$ has the properties

(i) If $x \in \mathfrak{D}(A(a)) \cap \mathfrak{D}(A(b))$ then $x \in \mathfrak{D}(A(s a+t b))$ and $A(s a+t b) x=$ $s A(a) x+t A(b) x$.

(ii) If $x \in \mathfrak{D}(A(a) A(b)) \cap \mathfrak{D}(A(b) A(a))$ then $x \in \mathfrak{D}(A([a, b]))$ and $A([a, b]) x=A(a) A(b) x-A(b) A(a) x$.

II. The function $a \rightarrow A^{*}(a)$ has the properties

(i) If $x^{*} \in \mathfrak{D}\left(A^{*}(a)\right) \cap \mathfrak{D}\left(A^{*}(b)\right)$ then $x^{*} \in \mathfrak{D}\left(A^{*}(s a+t b)\right)$ and $A^{*}(s a+t b) x^{*}=s A^{*}(a) x^{*}+t A^{*}(b) x^{*}$.

(ii) If $x^{*} \in \mathfrak{D}\left(A^{*}(a) A^{*}(b)\right) \cap \mathfrak{D}\left(A^{*}(b) A^{*}(a)\right)$ then $x^{*} \in \mathfrak{D}\left(A^{*}([a, b])\right)$ and $A^{*}([a, b]) x^{*}=A^{*}(b) A^{*}(a) x^{*}-A^{*}(a) A^{*}(b) x^{*}$.

Recalling that if a sequence of once continuously differentiable functions and the sequences of first order derivatives converge uniformly on some domain then the limit function is once continuously differentiable and its partial derivatives are the limits of the sequences of partial derivatives, we have, using (3) and Theorem 1,

Theorem 5. If $y \in \cap{ }_{\jmath=1} \mathfrak{D}\left(A\left(e_{j}\right)\right)$ then $T(p) y$ is once continuously differentiable in some neighbourhood, in $\pi$, of the origin and (3) holds. Conseguently, $T(p) y \in \mathfrak{D}(A(a))$ for $a \in E_{n}$ and $p$ in this neighbourhood.

The following theorem is an immediate consequence of Theorem 2.

Theorem 6. If $y \in \bigcap_{k=1}^{n \mathfrak{D}}\left(A\left(e_{k}\right)\right) \cap \mathfrak{D}\left(A\left(e_{i}\right) A\left(e_{j}\right)\right.$ then $y \in \mathfrak{D}\left(A\left(e_{j}\right) A\left(e_{i}\right)\right)$.

\section{REFERENCES}

1. E. Hille, Lie theory of semi-groups of linear transformations, Bull. Amer. Math. Soc., 56 (1950).

2. E. Hille and R. S. Phillips, Functional analysis and semi-groups, Amer. Math. Soc. Coll. Publ., 31 (1957).

3. K. de Leeuw, On the adjoint semi-group and some problems in the theory of approximation, Math. Zeit., 73 (1950).

Yale University 